\title{
VARIATIONAL ITERATION METHOD FOR CONSERVATIVE OSCILLATORS WITH COMPLICATED NONLINEARITIES
}

\author{
Y. M. Chen ${ }^{1 *}$, G. Meng ${ }^{1}$, J. K. Liu ${ }^{2}$ \\ ${ }^{1}$ State Key Laboratory of Mechanical System and Vibration \\ Shanghai Jiao Tong University, Shanghai 200240, China \\ ${ }^{2}$ Department of Mechanics, Sun Yat-sen University \\ Guangzhou 510275, China \\ *Corresponding author: Y.M. Chen, chenyanmao@hotmail.com
}

\begin{abstract}
The variational iteration method is employed to solve conservative oscillator containing complicated nonlinearities. In order to expand the nonlinear terms into truncated Fourier series, an approach of undetermined coefficient is proposed. Numerical examples shows the feasibility and efficiency of the variational iteration method as well as the presented technique for Fourier series expansion.
\end{abstract}

Keywords- Variational iteration method, complicated nonlinearity, Fourier series expansion

\section{INTRODUCTION}

Recent years have witnessed the wide applications of the variational iteration method (VIM), initiated by He [1-3], in various nonlinear problems [4-9]. In this paper, we restrict our attention to the VIM for solving nonlinear oscillating equations with very complicated nonlinearities. Since the early stage of its development, the VIM has been extensively employed to address nonlinear oscillators, e.g., conservative oscillators such as vibrations of the eardrum [3], the Duffing equation [3, 11], the ball-bearing oscillator [6-7], the mathematic pendulum [3, 6-7,10], nonlinear oscillators with discontinuities [12] and that with fractal force [13], and so on.

Since the VIM is usually implemented in the frequency domain, for nonlinear oscillators with complicated functions, a big obstacle will be confronted when expanding the nonlinearities into truncated Fourier series. To this end, an undetermined coefficient method is proposed to tackle this difficulty. With the help of this method, the VIM solutions are obtained to very high accuracy.

\section{VARIATIONAL ITERATION METHOD}

Consider a nonlinear conservative oscillator described as

$$
\ddot{x}+f(x)=0, x(0)=A, \quad x(0)=0,
$$

where the superscript denotes the differentiation with respect to time $t, A$ is a given constant, and $f(x)$ is a complex function. Equation (1) can be rewritten as 


$$
\ddot{x}+\omega^{2} x+x+f(x)-\omega^{2} x:=\ddot{x}+\omega^{2} x+F(\omega, x)=0, \quad x(0)=A, \quad \dot{x}(0)=0,
$$

where $\omega$ is the priori unknown frequency of the periodic solution $x(t)$ being sought.

Using He's variational iteration method, an iteration scheme is usually given as

$$
x_{n+1}=x_{n}+\frac{1}{\omega_{n}^{2}} \int_{0}^{\tau} \sin (s-\tau)\left[\omega_{n}^{2} x_{n}^{\prime \prime}+x_{n}+f\left(x_{n}\right)\right] d s, n=0,1,2, \cdots
$$

subject to the initial conditions as

$$
x_{n}(0)=A, \quad x_{n}^{\prime}(0)=0,
$$

where the superscript denotes the differentiation with respect to $\tau$. Assume that the input of starting function has the form

$$
x_{0}(\tau)=A \cos \tau
$$

The $n$ th-order VIM approximations for $\omega$ is determined by eliminating the secular term, i.e., solving the equation

$$
\int_{0}^{2 \pi} \cos (s)\left[\omega_{n}^{2} x_{n}^{\prime \prime}+f\left(x_{n}\right)\right] d s
$$

\section{FOURIER SERIES EXPANSION OF $f\left(x_{n}\right)$}

The major difficulty in obtaining Eq. (6) is expanding the nonlinear terms into Fourier series. For example, a nonlinear function such that $1 / \sqrt{1-x^{2}(\tau)}$ has to be transformed into truncated Fourier series of $\tau$, in which $x$ is truncated Fourier series containing $N$ harmonics. In most literature, the harmonic balance equation can be easily obtained because the considered nonlinearities are described as integral power functions. An omnipotence yet inefficient method of obtaining the Fourier coefficients is integrating the product of $1 / \sqrt{1-x^{2}(\tau)}$ and each harmonic in $\tau \in[0,2 \pi]$. However, it is tough even impossible to use this technique in our study.

Take a functional $f(\tau)=h(x(\tau))$ of $\tau$ to illustrate a new method of undetermined coefficients for Fourier series expansion. Note that $x(\tau)$ is a truncated Fourier series, hence $f(\tau)$ has a period as $T=2 \pi$. In the cyclic method [14], first a set of points on the periodic orbit at $M$ uniformly distributed time levels such that $\left\{t_{n}=2(n-1) \pi / M, n=1,2, \cdots, M\right\}$ is selected. At the chosen time points, $f(\tau)$ can be discretized as 


$$
\mathbf{f}=\left\{f\left(\tau_{n}\right)\right\}^{\mathrm{T}}=\left\{h\left(z\left(\tau_{n}\right)\right)\right\}^{\mathrm{T}} .
$$

Here $\mathbf{f}$ is a constant vector. Assume $f(\tau)$ has a truncated Fourier series as

$$
f(\tau)=\sum_{k=0}^{N}\left(c_{k} \cos k \tau+s_{k} \sin k \tau\right)
$$

where $c_{k}$ and $s_{k}$ are priori unknowns. According to Eqs. (7) and (8), at the uniformly selected points $\left\{\tau_{n}=2(n-1) \pi / M, n=1,2, \cdots, M\right\}$, one has

$$
\sum_{k=0}^{N}\left(c_{k} \cos k \tau_{n}+s_{k} \sin k \tau_{n}\right)=\mathbf{f}_{n}, n=1,2, \cdots, M .
$$

Let $\mathbf{q}=\left\{c_{0}, c_{1}, s_{1}, \cdots, c_{N}, s_{N}\right\}^{\mathrm{T}}$, one can deduce a linear equation

$$
\mathbf{H q}=\mathbf{f}
$$

where $\mathbf{H}$ is a matrix of dimension $M \times(2 N+1)$ with the row vectors as

$$
\mathbf{H}_{n}=\left[\begin{array}{llllll}
1 & \cos \tau_{n} & \sin \tau_{n} & \cdots & \cos N \tau_{n} & \sin N \tau_{n}
\end{array}\right], \quad n=1,2, \cdots, M .
$$

In order to determine $\mathbf{q}$, theoretically there must be $2 N+1$ chosen time points, i.e., $M=2 N+1$. However, choosing only $2 N+1$ points is not enough for ensuring high accuracy of q. If more than $2 N+1$ points are adopted, i.e., when $M>2 N+1$, Eq. (10) becomes over-determined. The least squares method is employed to obtain $\mathbf{q}$ of the over-determined equations.

\section{NUMERICAL EXAMPLES}

Consider the following oscillating equation [15]

$$
\ddot{x}+x / \sqrt{1+x^{2}}=0, \quad x(0)=A, \dot{x}(0)=0
$$

Figure 1 shows a phase plane of Eq. (12) with $A=10$. The solution provided by the VIM is in excellent agreement with the numerical result. The frequency attained by the VIM versus $A$ is plotted in Fig. 2. For any given value of $A$, it holds that $-A \leq x \leq A$. Since $\sqrt{1+x^{2}}$ approaches 1 when $A<<1$, Eq. (12) becomes a harmonic oscillator. Therefore, the angular frequency converges 1 as $A$ approaches 0 , as shown in Fig. 2.

The second example corresponds to the following equation

$$
\ddot{x}+f(x)=0, f(x)=\left\{\begin{array}{l}
x+\sigma, x \geq 0 \\
x-\sigma, x<0
\end{array}, \quad x(0)=A, \quad \dot{x}(0)=0\right.
$$

where $\sigma$ is a positive constant. Equation (13) describes a discontinuous nonlinear oscillator. The phase planes for different values of $\sigma$ are plotted in Fig. 3. When 
$\sigma=0.1$, the phase plane is almost the same as a cycle with the diameter as 1 . That is because when $\sigma<<1$, Eq. (13) can be approximately considered as a harmonic oscillator. As $\sigma$ increases, the velocity increases and the phase plane become complicated. Even though, the VIM is capable of tracking the complex responses. Also, one can see from Fig. 4 the angular frequency decreases as $A$ increasing, while it increases as $\sigma$ increasing, respectively.

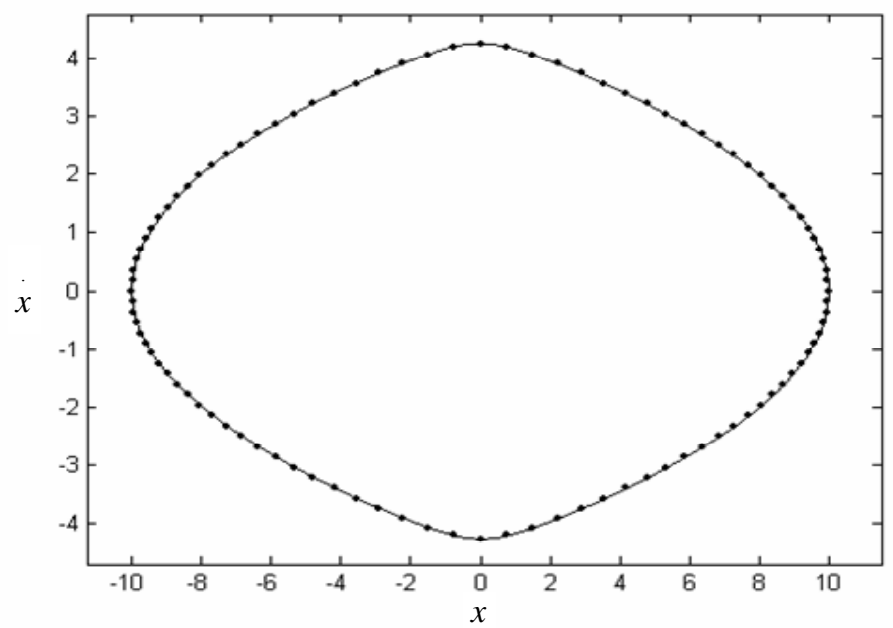

Fig. 1 Phase plane of Eq. (12) with $A=10$, where solid line denotes numerical solution and heavy dots the 10th-order VIM approximation with $N=10$

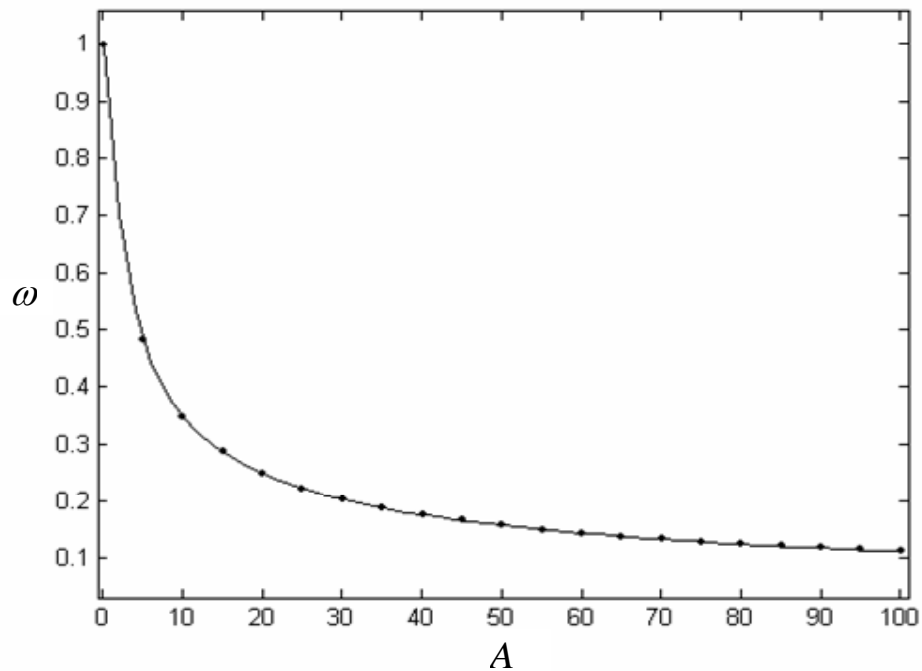

Fig. 2 Angular frequency of Eq. (12) versus A, where solid line denotes numerical solution and heavy dots the 10th-order VIM approximation with $N=10$ 


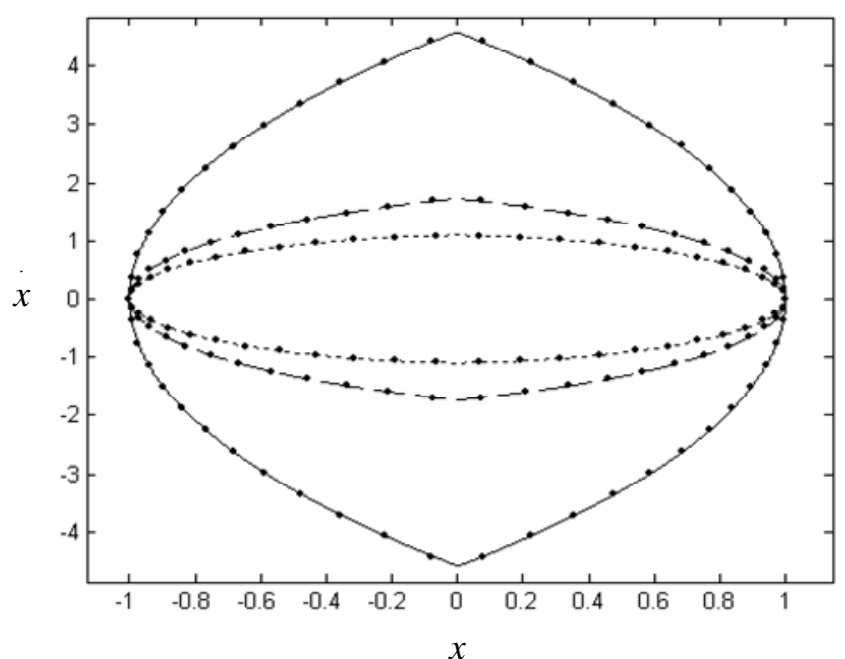

Fig. 3 Phase planes for Eq. (13) with $A=1$, where the heavy dots denote the 30th-order VIM approximation with $N=30$, the dot line for $\sigma=0.1$, the dashed line for $\sigma=1$ and the solid line for $\sigma=10$.

In the last example, we consider

$$
\ddot{x}+\operatorname{sgn}(x) / \sqrt{1+x^{2}}=0,, \quad x(0)=A, \quad x(0)=0, \operatorname{sgn}(x)= \begin{cases}1, & x>0 \\ 0, & x=0 \\ -1, & x<0\end{cases}
$$

The nonlinear restoring force $f(x)=\operatorname{sgn}(x) / \sqrt{1+x^{2}}$ versus displacement $x$ is shown in Fig. 5. This nonlinear function is discontinuous and is complicated than bilinear term of Eq. (13). The phase plane and response-frequency curve also show good excellent agreement of the VIM results and the numerical ones. The phase plane even contains discontinuous points, i.e., the points when the velocity takes extreme values.
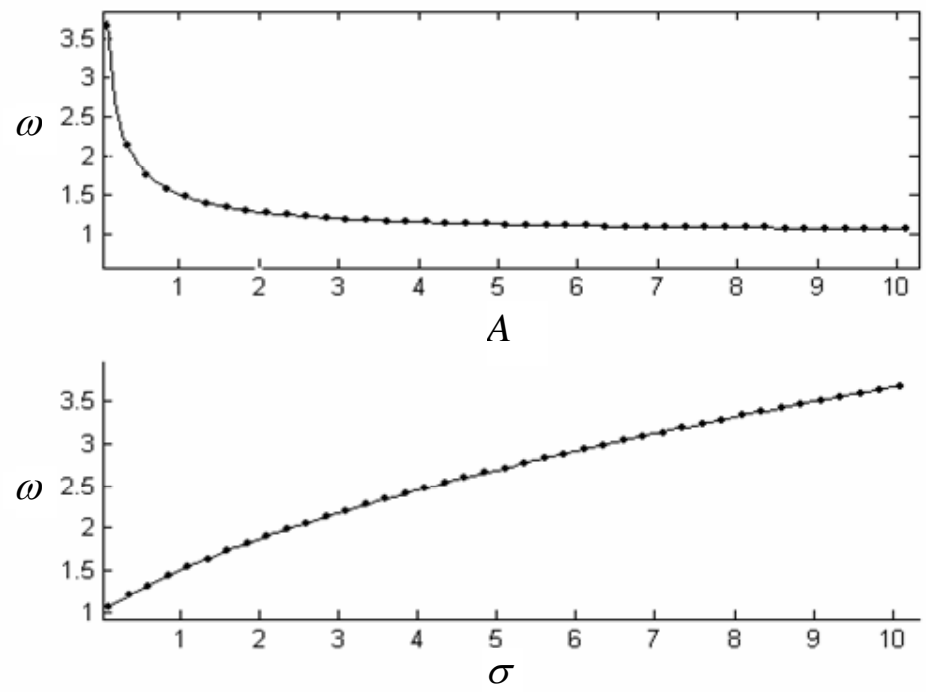

Fig. 4 Angular frequency of Eq. (13) versus $A$ and $\sigma$, respectively, where solid line denotes numerical solutionS and heavy dots the 30th-order VIM approximation, $N=30$ 


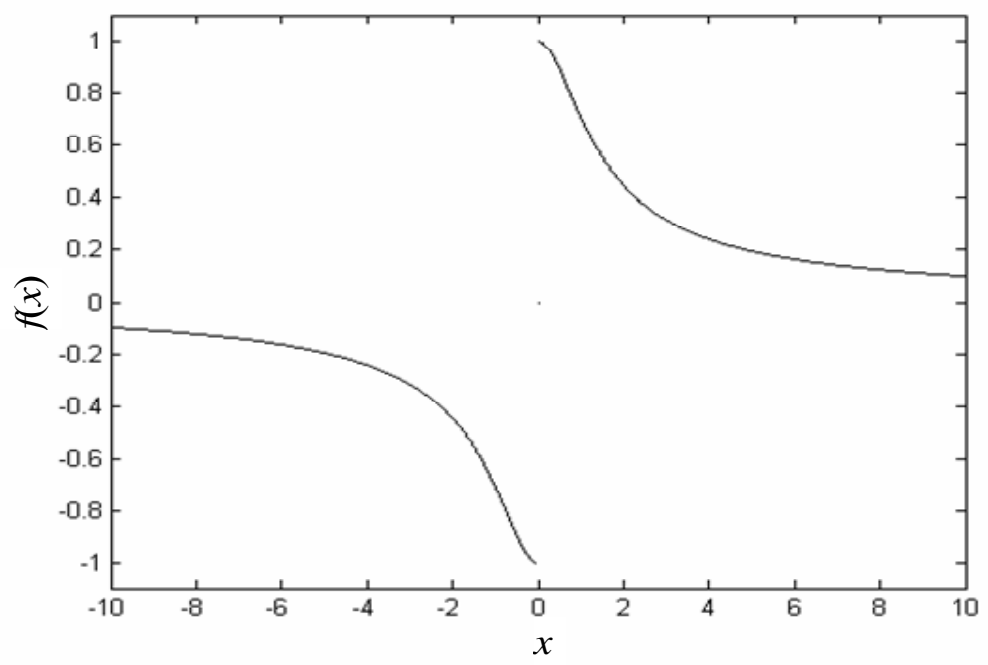

Fig. 5 The nonlinear term in Eq. (14) versus $x$

\section{CONCLUSIONS}

We have employed the VIM to solve autonomous oscillators with complex nonlinearities. An approach is also proposed, based on the cyclic method, to expand the nonlinear terms into time Fourier series. This approach makes it easy to implement the VIM to seek high order iteration approximations. Three numerical examples are presented to validate the proposed approaches. The solutions attained by the VIM, together with the presented approach of Fourier series expansion, are in excellent agreement with the numerical results. Basically, the approximations can be obtained to any desired accuracy without any additional difficulty.

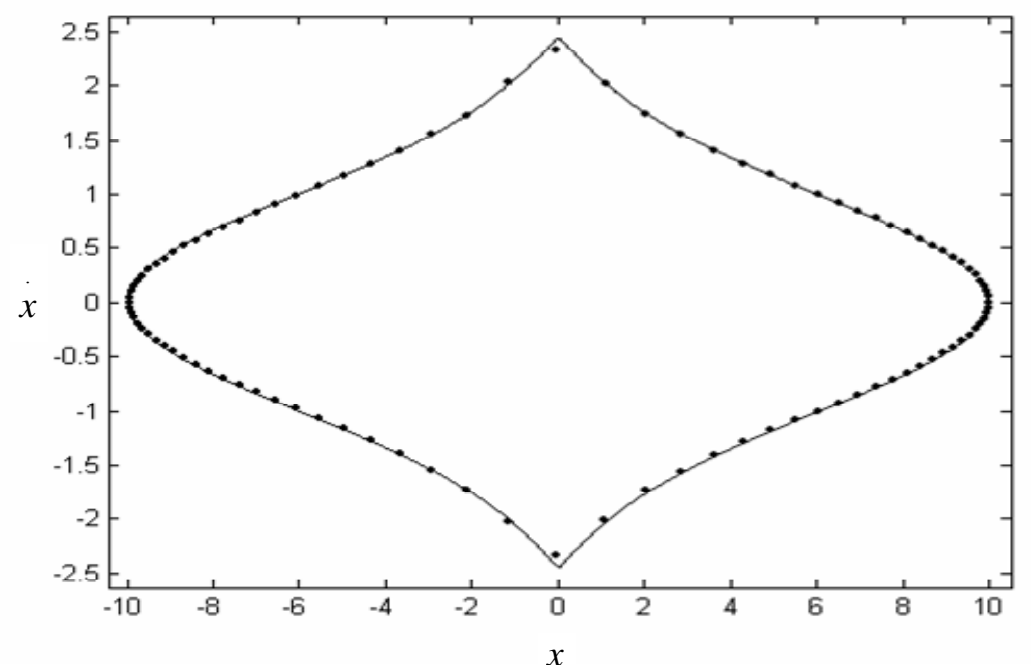

Fig. 6 Phase plane for Eq. (14) with $A=10$, where the heavy dots denote the 30th-order VIM approximation with $N=50$ and the solid line for the numerical solution 


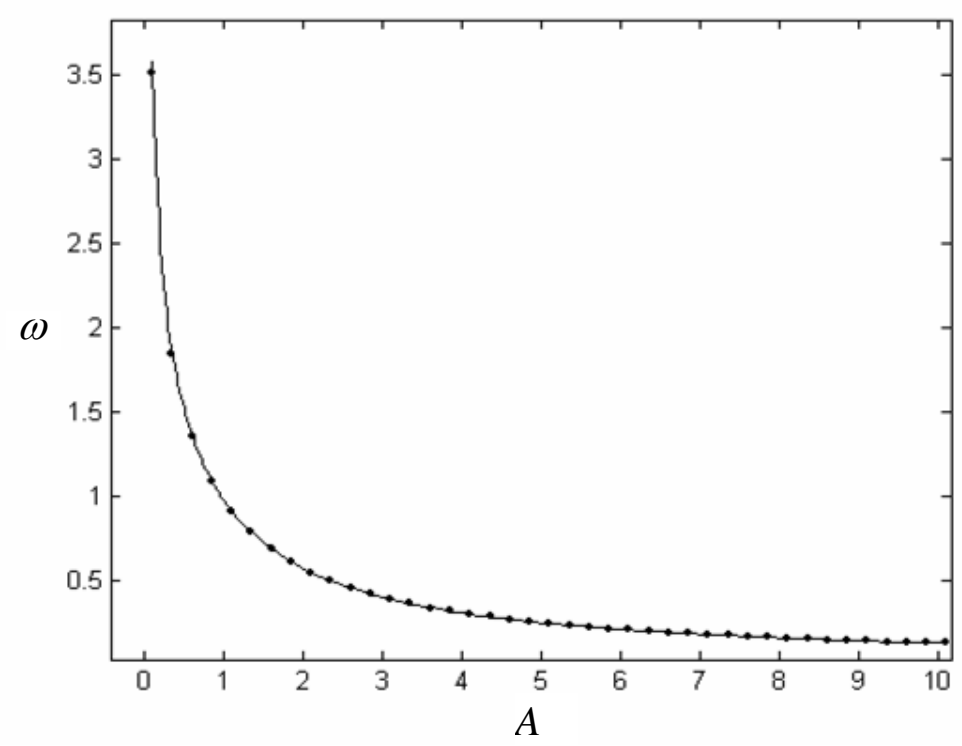

Fig. 7 Angular frequency of Eq. (14) versus $A$, where solid line denote numerical solution and heavy dots the 30th-order VIM approximation with $N=50$.

\section{ACKNOWLEDGEMENT}

This work is supported by the National Natural Science Foundation of China (10772202, 10102023, 10972241), Doctoral Program Foundation of Ministry of Education of China (20090171110042), China Postdoctoral Science Foundation (20090460628) and Shanghai Postdoctoral Science Foundation (10R21413600).

\section{RERERENCES}

1. J. H. He, Approximate analytical solution for seepage flow with fractional derivatives in porous media, Computer Methods Applied Mechanics in Engineering 167, 57-68, 1998.

2. J. H. He, Approximate solution of nonlinear differential equations with convolution product nonlinearities, Computer Methods Applied Mechanics in Engineering 167, 69-73, 1998.

3. J. H. He, Variational iteration method-a kind of non-linear analytical technique: some examples, International Journal of Non-linear Mechanics 34, 699-708, 1999.

4. J. H. He, Variational iteration method for autonomous ordinary differential systems, Applied Mathematics and Computation 114, 115-123, 2000.

5. S. Momani, S. Abuasad and Z. Odibat, Variational iteration method for solving nonlinear boundary value problems, Applied Mathematics and Computation 183, 1351-1358, 2006.

6. J. H. He, Variational iteration method-Some recent results and new interpretations, Journal of Computational and Applied Mathematics 207, 3-17, 2007.

7. J. H. He and X. D. Wu, Variational iteration method: New development and applications, Computers Mathematics with Applications 54, 881-894, 2007. 
8. J. J. Ramos, On the variational iteration method and other iterative techniques for nonlinear differential equations, Applied Mathematics and Computation 199, 39-69, 2008.

9. M. Rafei, H. Daniali and D. D. Ganji, Variational iteration method for solving the epidemic model and the prey and predator problem, Applied Mathematics and Computation 186, 1701-1709, 2007.

10. V. Marinca, N. Herişanu and C. Bota, Application of the variational iteration method to some nonlinear one-dimensional oscillations, Meccanica 43, 75-79, 2008 .

11. V. Marinca and N. Herişanu, Periodic solutions of Duffing equation with strong non-linearity, Chaos, Solitons and Fractals 37, 144-149, 2008.

12. M. Rafei, D. D. Ganji, H. Daniali and H. Pashaei, The variational iteration method for nonlinear oscillators with discontinuities, Journal of Sound and Vibration 305, 614-620, 2007.

13. T. Öziş and A. Yıldırım, A study of nonlinear oscillators with $u^{1 / 3}$ force by He's variational iteration method, Journal of Sound and Vibration 306, 372-376, 2007.

14. P. S. Beran and D. J. Lucia, A reduced order cyclic method for computation of limit cycles, Nonlinear Dynamics 39, 143-158, 2005.

15. D. H. Shou and J. H. He, Application of parameter-expanding method to strongly nonlinear oscillators. International Journal of Nonlinear Sciences and Numerical Simulation, 8(1): 121-124, 2007. 